\title{
Digital twinning within a novel human-in-the-loop verification method for HUD safety-critical approach and landing
}

Paul Wisely

Paul Wisely, "Digital twinning within a novel human-in-the-loop verification method for HUD safety-critical approach and landing," Proc. SPIE 11759, Virtual, Augmented, and Mixed Reality (XR) Technology for Multi-Domain Operations II, 117590H (12 April 2021); doi: 10.1117/12.2585318 


\title{
Digital twinning within a novel human-in-the-loop verification method for HUD safety-critical approach and landing
}

\author{
Paul Wisely ${ }^{\mathrm{a}}$ \\ aAardvark Aerospace Ltd., 2 Jubilee Villas, Rock Hill Road, Egerton, Kent TN27 9DP, United \\ Kingdom
}

\begin{abstract}
We have designed a visual flight guidance system that enables manual control of aircraft operations in degraded visual environments down to Cat III, both for take-off and landing.

This has been achieved by means of visual guidance cues displayed on a head up display (HUD) and whereas this is not in itself novel, our development methods and approach to verifying its operation we believe are.

In order to certify the system as airworthy, compliance with the relevant airworthiness standards defined in 14 CFR part 21 and other related guidance material, needs to be demonstrated. Demonstrating compliance by actually flying the aircraft a statistically appropriate number of times is prohibitively expensive.

The challenge in this case was to harmonize existing flight guidance algorithms with a faithful aero model of a new airframe and to demonstrate their effectiveness in a manner that was economically viable.

To achieve this, we constructed a Digital Simulation and Verification Environment (DSVE) that hosted a Digital Twin (DT) of the system such that its operation in real conditions could be accurately predicted.
\end{abstract}

\section{Introduction}

We have designed a visual flight guidance system that enables manual control of aircraft operations in degraded visual conditions both for take-off and landing in environments down to Cat III a.

This has been achieved by means of visual guidance cues, computed from aircraft sensor data, displayed on a head up display (HUD) and whereas this is not in itself novel, our development methods and approach described in this paper to verify its operation we believe in many respects are.

In order to certify the system as airworthy, compliance with the relevant airworthiness standards defined in 14 CFR part 21 and other related guidance material, needs to be demonstrated to the satisfaction of the authorities.

The challenge in this case was to harmonize existing flight guidance algorithms with a faithful aero model of a new airframe and to demonstrate their effectiveness in a manner that is economically viable.

To achieve this, we constructed a system called a Digital Simulation and Verification Environment (DSVE) that hosted a Digital Twin (DT) of the system such that its operation in real conditions could be accurately predicted and hence its fitness for purpose verified.

The design approach for the digital twin was to host the guidance algorithms, developed by Hoh Aeronautics Inc. of Lomita California and implemented using the model-based implementation techniques of Ansys SCADE Suite and SCADE Display, to replicate the flight guidance symbology, together with an aerodynamic model of the aircraft developed by Laminar Research Inc, using blade element theory.

\section{Purpose of the Flight Guidance System.}

The guidance system in this application is constructed such that it provides a replication of the head down instrumentation information together with additional specific flight guidance information to the pilot by means of projection on a Head Up Display (HUD) Combiner located in the pilot's forward field of view as shown in figure one. This enables the pilot to fly an instrument approach solely by reference to the HUD.

Whereas the HUD provides similar information to that available head down, flying with a HUD is superior in several respects:

Virtual, Augmented, and Mixed Reality (XR) Technology for Multi-Domain Operations II, edited by Mark S. Dennison,

David M. Krum, John (Jack) N. Sanders-Reed, Jarvis (Trey) J. Arthur III, Proc. of SPIE Vol. 11759, 117590H

(C) 2021 SPIE · CCC code: $0277-786 X / 21 / \$ 21 \cdot$ doi: $10.1117 / 12.2585318$

Proc. of SPIE Vol. 11759 117590H-1 
- Firstly, the displayed information is conformal, that is to say objects depicted on the display overlay the realworld objects that they represent when appropriate.

- Secondly, the display is collimated, that is to say effectively focussed at optical infinity. For technical and human factors reasons, the focus is of course short of true infinity to ensure that all points within the binocular disparity field are convergent.

- Thirdly, the viewing angles and scale of the display are larger than those subtended head down which makes the display easier to use by the operator.

- Finally, the pilot can maintain situational awareness more readily when flying "eyes out" for the approach and is thus focussed to acquire the real-world touchdown zone.

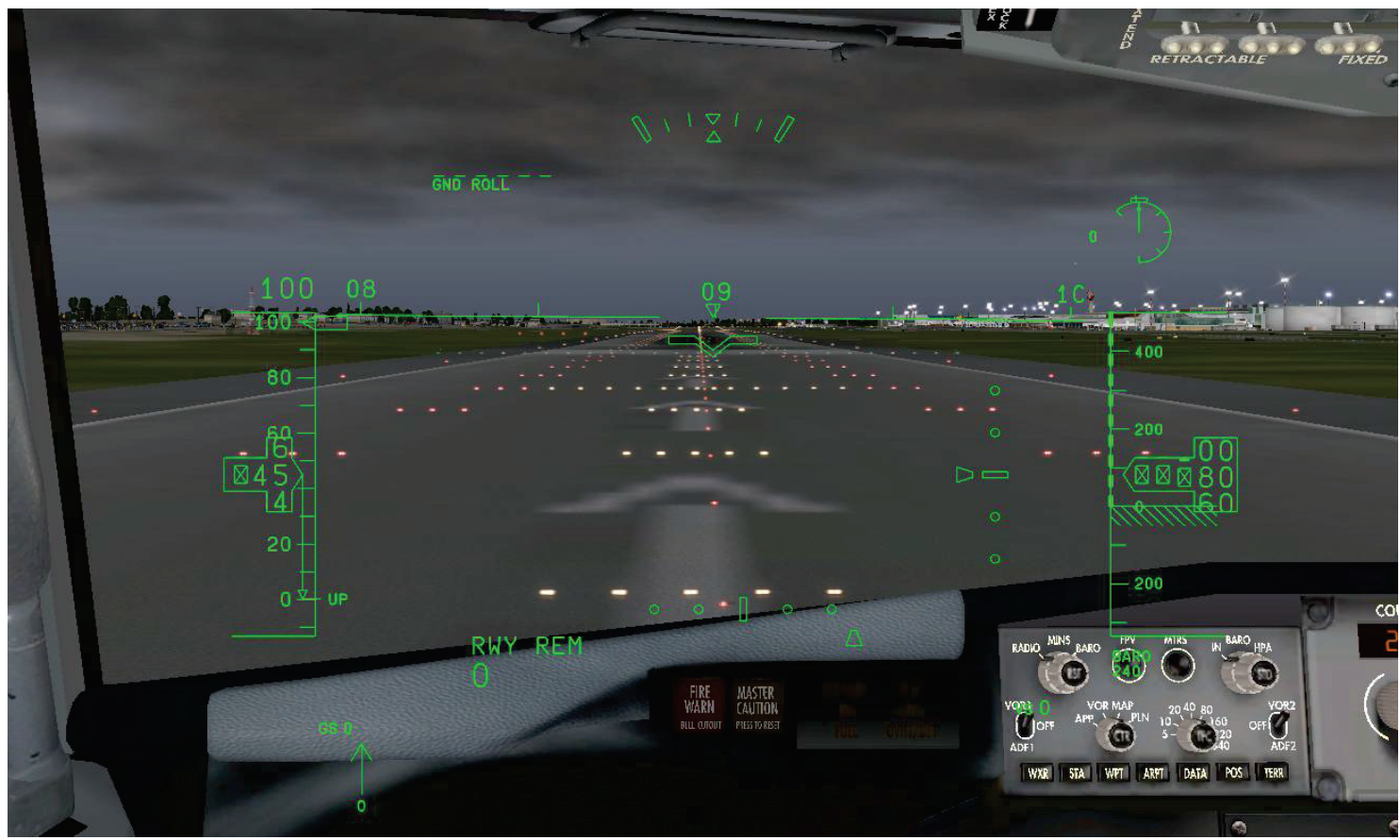

Figure one: General view through the windshield

\section{The Requirements}

In order to be certified for commercial airworthiness, systems must be shown to comply with the appropriate regulations. For low visibility operations to Cat III a, these are defined in FAA advisory circular AC 120 28D appendices 2 and 3. This document relates to the on-board and off-board equipment used during take-off and landing and the demonstration of the accuracy and integrity requirements of the combined equipments.

The type certification approval for the equipment, system installations and test methods should be based upon a consideration of factors such as the intended function of the installed system, its accuracy, reliability, and fail-safe features.

Landing System Performance for low visibility landings systems shall be demonstrated to achieve the accuracy of performance with the probabilities defined as follows:

- Longitudinal touch down earlier than a point on the runway $200 \mathrm{ft}$. from the threshold to a probability of $10^{-6}$

- Longitudinal touchdown beyond $2700 \mathrm{ft}$. from threshold to a probability of $10^{-6}$

- Lateral touchdown with the outboard landing gear more than $70 \mathrm{ft}$. from runway centreline to a probability of $10^{-6}$

- Structural limit load, to a probability of $10^{-6}$ 
- An acceptable means of establishing that the structural limit load is not exceeded is to show separately and independently that the limit load that results from a sink rate at touchdown is not greater than 10 f.p.s.

- Bank angle resulting in hazard to the airplane to a probability of $10^{-7}$

- A hazard to the airplane is interpreted to mean a bank angle resulting in any part of the wing, high lift device or engine nacelle touching the ground

- Also, specific precision must be met on approach as follows:

○ Glideslope tracking between 700 and 100 feet; the greater of $\pm 35 \mu \mathrm{A}$

○ Localiser tracking between 700 and $300 \mathrm{ft} ; \pm 35 \mu \mathrm{A}$

○ Localiser tracking between 300 and $100 \mathrm{ft} ; \pm 25 \mu \mathrm{A}$

○ Airspeed tracking between 700 and $50 \mathrm{ft} ; \pm 5$ knots

\section{System Operation}

The facility to provide manual flight guidance by means of a HUD in a commercial aircraft is not novel [1].

Guidance cues calculated by specialist proprietary algorithms created and developed by Hoh Aeronautics ${ }^{1}$ have been Certified by the FAA for use in Cat III a conditions for a number of years on commercial aircraft ${ }^{2}$.

Our particular use of digital twinning is, we think, fairly novel (and therefore interesting) because it includes an operatorin-the-loop control task interposed between the digital twin of the aero model and the digital twin of the control laws themselves.

The system provided a flight simulation at realistic airports worldwide by means of the Laminar Research X Plane simulation system that included adjustable weather conditions.

The twin recorded data during the approach and landing to facilitate subsequent statistical analysis such that comparisons with the regulations could be performed.

\section{Twin Test Station Arrangement}

The basic arrangement of the station comprises a computer, display screen, and twin inceptors to control the simulation operation.

The computer hosts several functions as follows:

○ X Plane 10 professional aircraft simulator which includes an aero model of the aircraft calculated through the use of blade element theory.

- With blade element theory, any surface, for example a wing, may be made up of several sections, one to four being typical, and each of these sections further divided into as many as ten separate subsections. The lift and drag of each section are then calculated, and the resulting effects applied to the whole aircraft.

- When this process is applied to each component, the simulated aircraft will fly in a manner that is very similar to its real-life counterpart.

- The Hoh Aeronautics control algorithms, implemented using a certified model-based software suite.

- A comprehensive HUD symbol set, again implemented by means of a model-based software suite. ${ }^{3}$

- An application providing a graphical user interface to enable the operator to enter and edit initial system parameters, manage the overlay of the HUD symbol set over the X Plane outside world scene, and record the necessary flight and touchdown data.

${ }^{1}$ Hoh aeronautics Inc. Palos Verdes Drive North, Lomita California

${ }^{2}$ Such as the Boeing 737 NG of American Airlines which is fitted with a BAE Systems HUD

${ }^{3}$ The model-based software suites are produced by Ansys and comprise SCADE Suite and SCADE Display 


\section{The Approach Control Task}

The approach symbology is illustrated in figure two

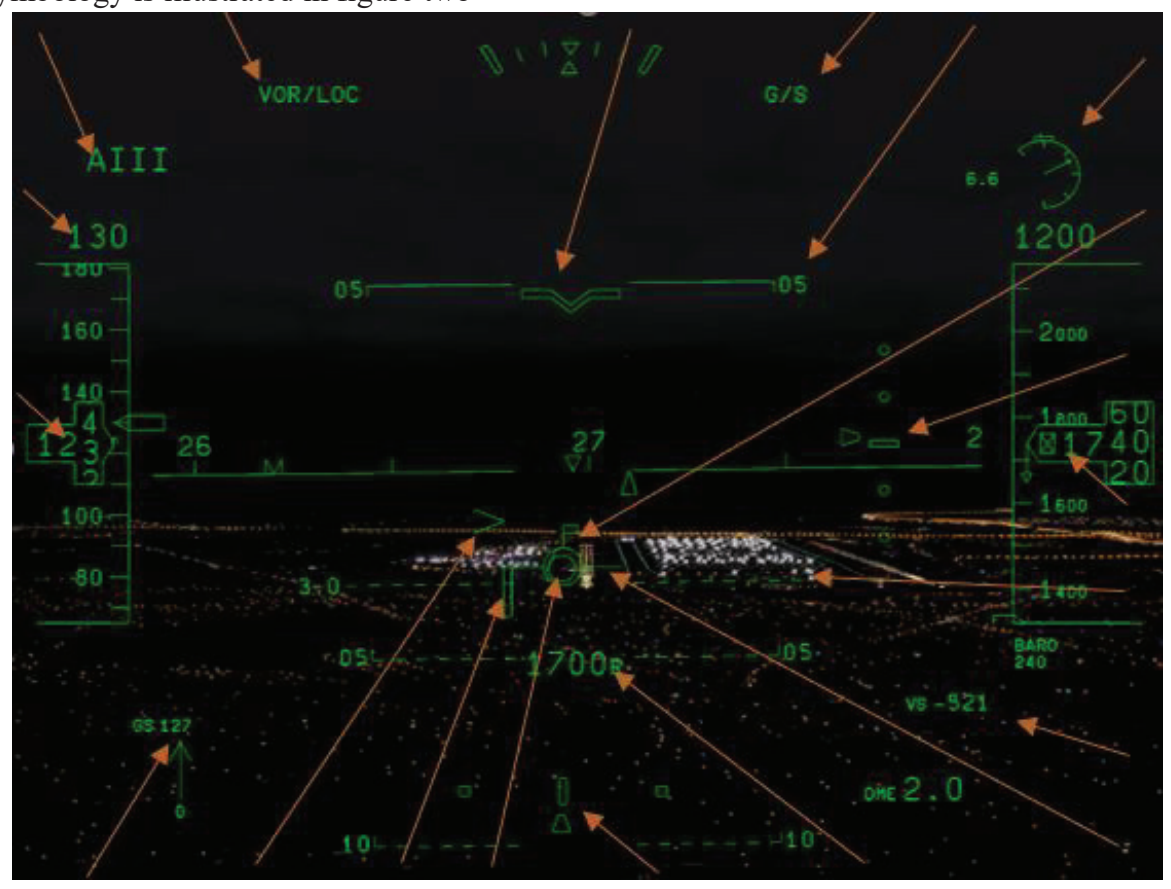

Figure two: approach symbol set

Taking the symbols indicated by the arrows from top left and working clockwise we have

\begin{tabular}{|c|c|c|}
\hline Symbol & Name & Comment \\
\hline AIII & Approach mode & Mode currently active \\
\hline VOR/LOC & Horizontal guidance mode engaged & \\
\hline Boresight & Aircraft Physical heading & \\
\hline $\mathrm{G} / \mathrm{S}$ & Vertical Guidance mode engaged & \\
\hline 05 & Pitch reference ladder & \\
\hline 6.6 & Angle of attack & \\
\hline Worm & Sideslip error & $\begin{array}{l}\text { Crab error indicated against the } \\
\text { vertical fin }\end{array}$ \\
\hline Scale & Glideslope deviation scale & \\
\hline 1740 & Current altitude & \\
\hline-3.0 & Desired glidepath & \\
\hline-521 & Vertical speed & \\
\hline Circle with wings & Flight path marker or velocity vector. & $\begin{array}{l}\text { Indicates the direction of the centre of } \\
\text { mass of the aircraft }\end{array}$ \\
\hline 1700 & Radar Altitude & \\
\hline Scale & Localiser deviation & \\
\hline Circle & Flight Director & \\
\hline Worm & Speed error worm & Error between desired and actual \\
\hline Chevron & Longitudinal Acceleration Caret & Indicates longitudinal state \\
\hline 127 & Groundspeed & \\
\hline 123 & Current airspeed & \\
\hline 130 & Selected Airspeed & Desired approach speed \\
\hline
\end{tabular}


The task is basically to fly so as to minimize indicated errors as follows:

The velocity vector is purely driven by aircraft/environment state and shows where the centre of mass of the airframe is going.

The flight director is driven by the horizontal/vertical guidance algorithms and indicates where the aircraft ought to be going, hence the task is to keep the symbols overlaying which indicates the approach is on track.

The speed error worm indicates the error between desired airspeed and the actual. The task here is to adjust the power to minimize the error worm to zero.

The longitudinal acceleration caret responds to power and for example would be set above the Velocity Vector wingtip if the worm indicates the need to speed up. When the airspeed is steady and on value, the acceleration caret will sit on the wingtip.

The crab angle error worm indicates whether slip or skid is occurring.

\section{Performing an Approach and Landing}

The operation is somewhat complex and is best appreciated through following an example of a test run with data capture and analysis as follows.

The airport selected for this example is London Heathrow, where an approach and landing is illustrated using runway 27L. HUD X plane switch-on initialisation pre MCDU data entry is shown in figure three.

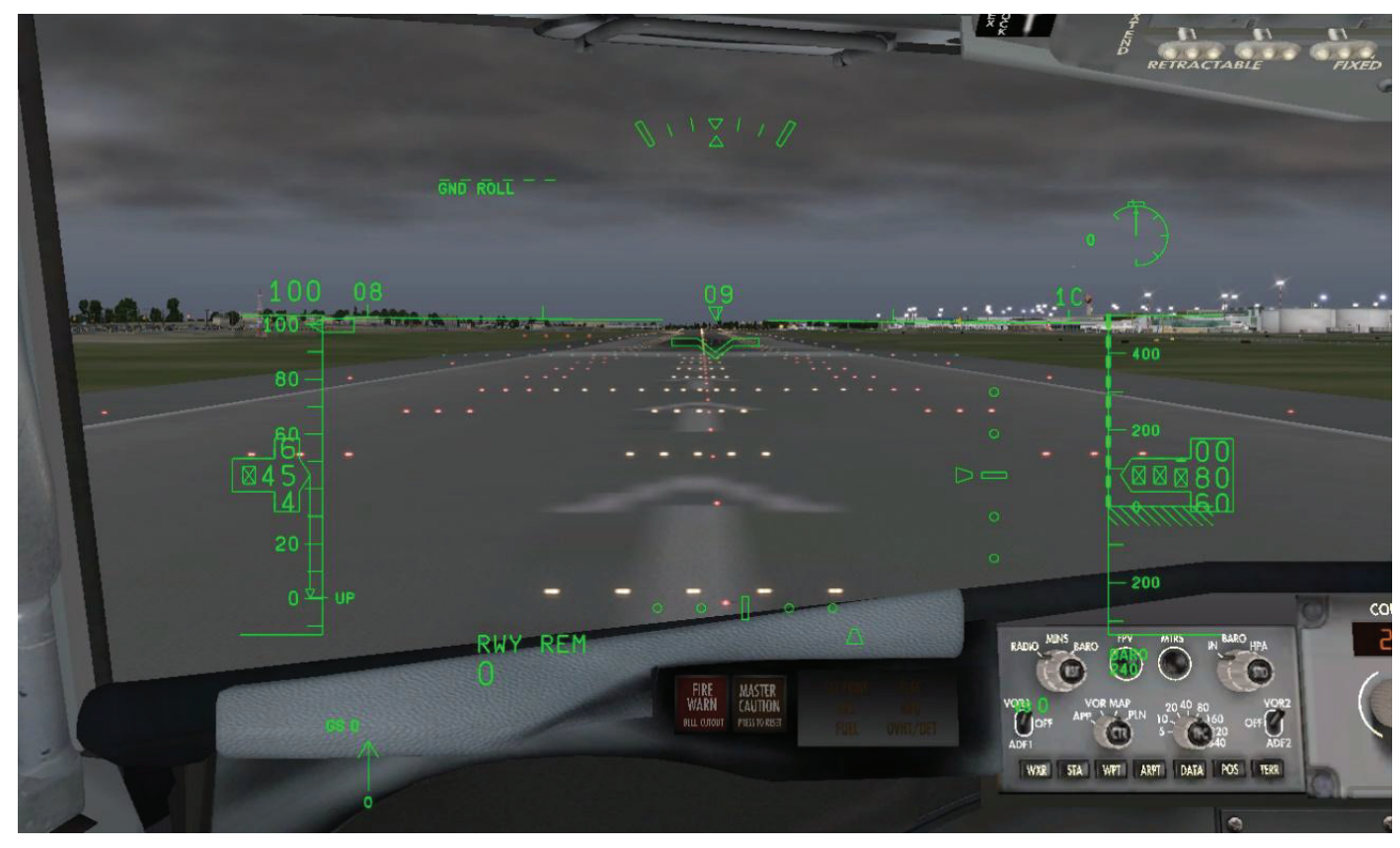

Figure three: X plane display with HUD Symbols on initialisation 
The approach data, desired glidepath, runway length and runway elevation were set as shown on the MCDU display in figure four, and airport details as shown in figure five.

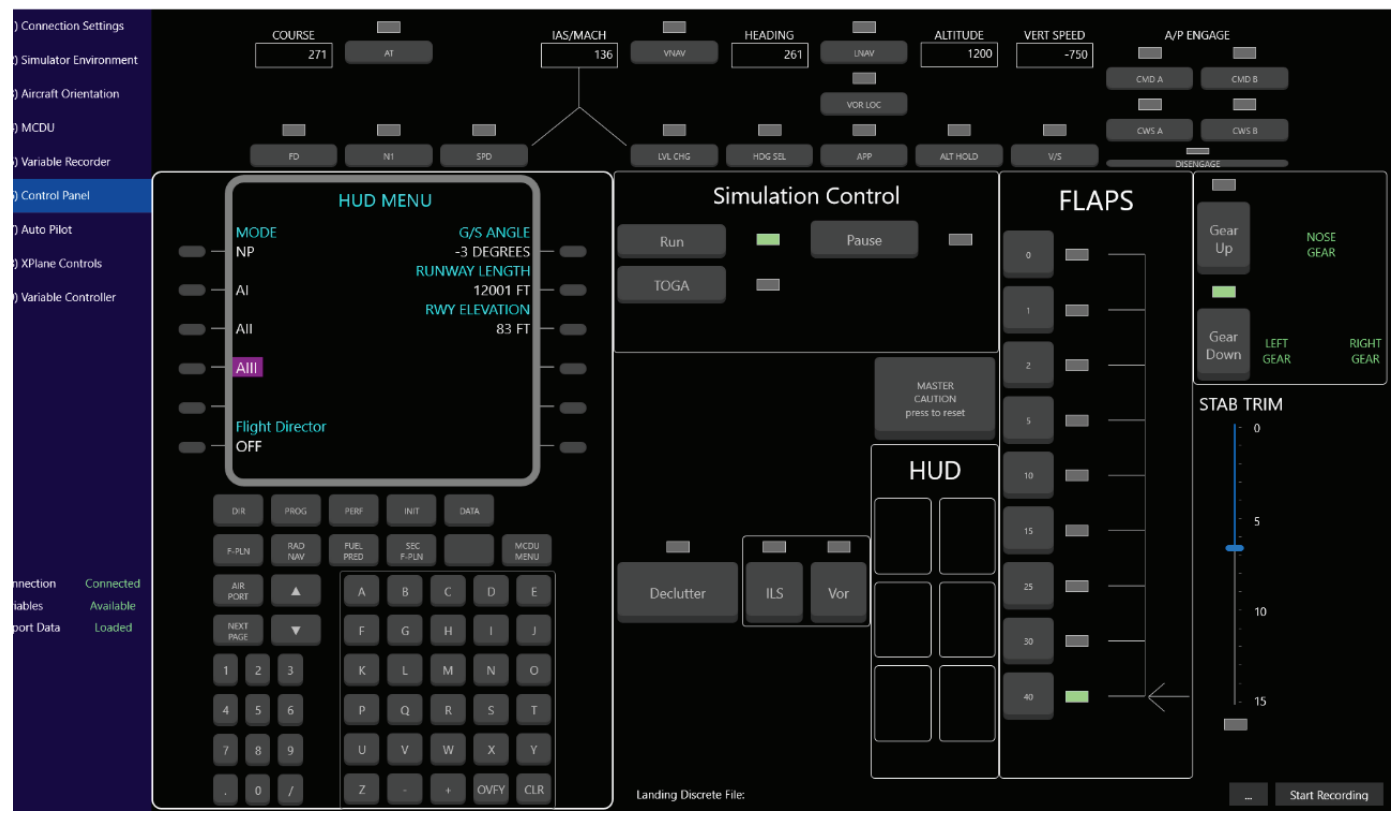

Figure four: Station Display containing autopilot control panel, flap settings, stabiliser trim, gear control and Multi Function Control and Display Unit (MCDU). This display is used to set up the desired approach

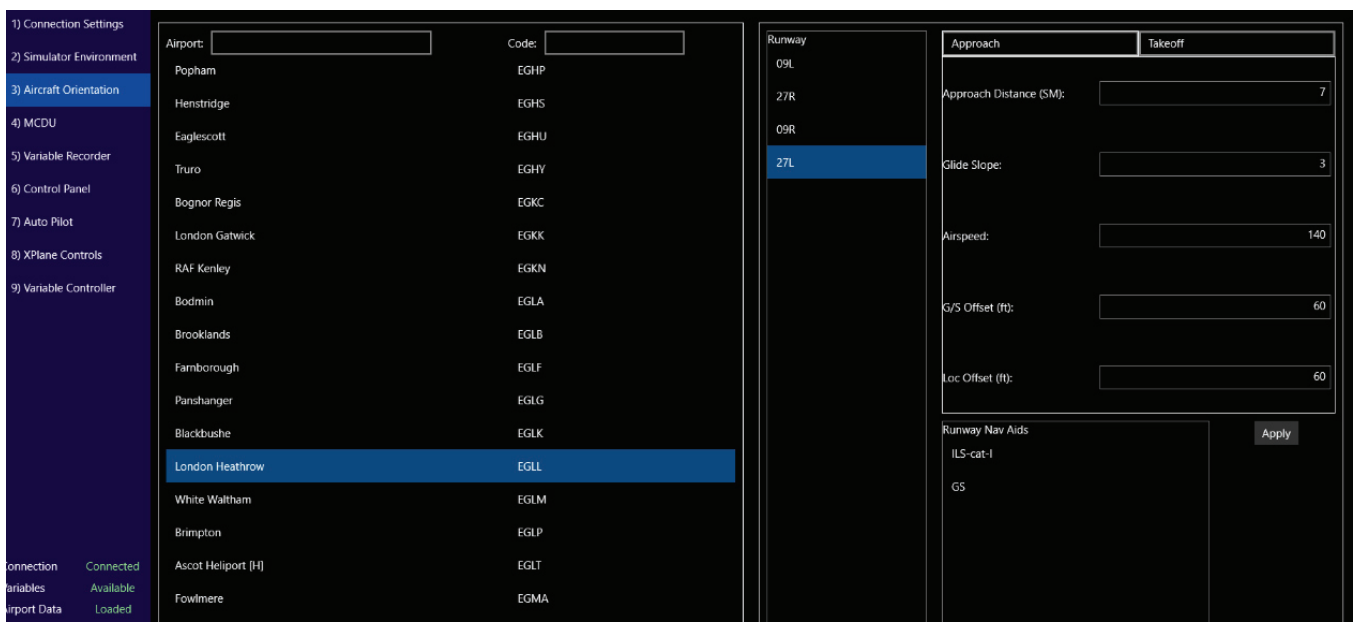

Figure five: Airport Control Panel

As data is input, the display updates until typically figure six. 


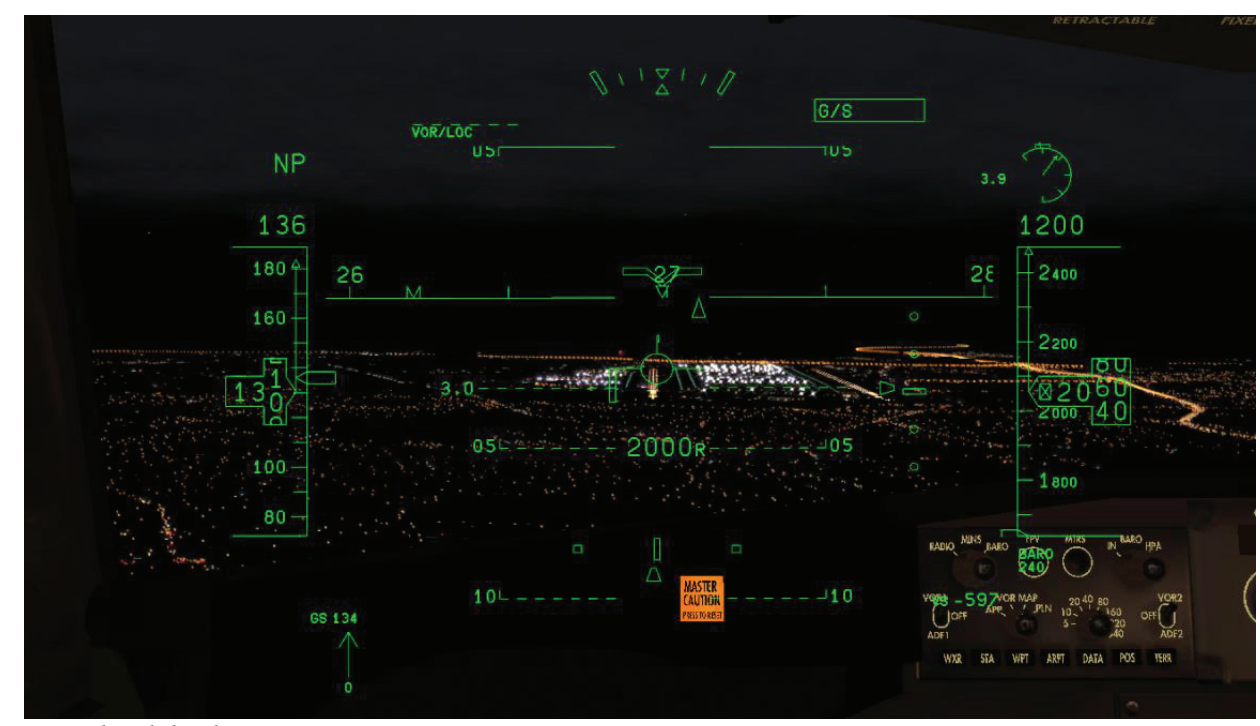

Figure six: initialised display

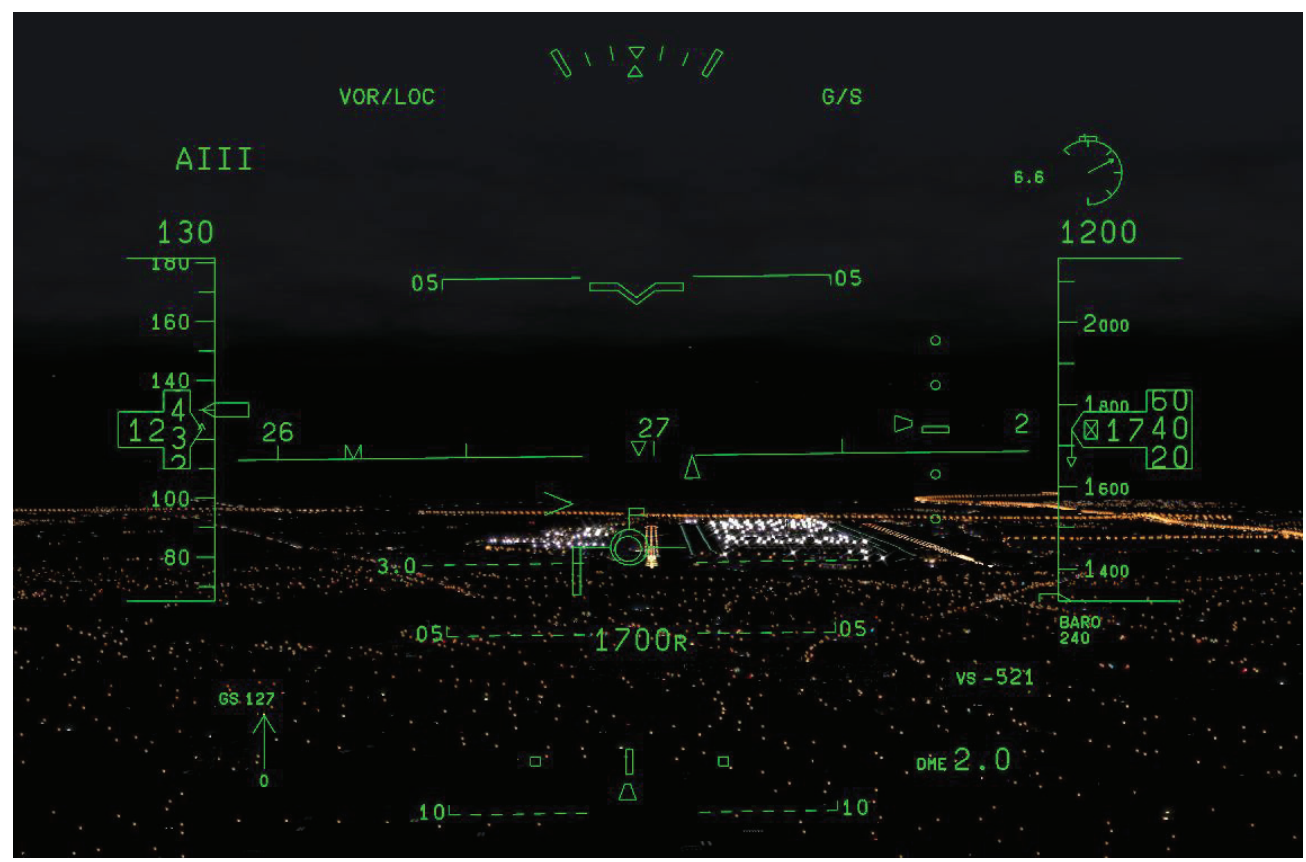

Figure seven: ILS capture and AII mode active

The system entered AIII mode automatically when conditions were right as controlled by the algorithms.

The approach was flown by adjusting the power to reduce the speed error worm on the wingtip to zero whilst keeping the Flight Path Marker (FPM) over the Flight Director (FD). This ensured correct ILS tracking and airspeed control. The aircraft was flown down to the flare, power retarded and the flare cue followed in a smooth movement until touchdown. The system detected touchdown and captured the point on the runway where this occurred together with other data.

Approaching touchdown, the vertical guidance entered a pitch only mode termed Flare Guidance which ensured a gentle touch down. 
Figure eight illustrates the aircraft tracking the Flare Guidance Cue and commands power reduction. The horizontal guidance enters a lateral only mode post touchdown to track the runway centreline.

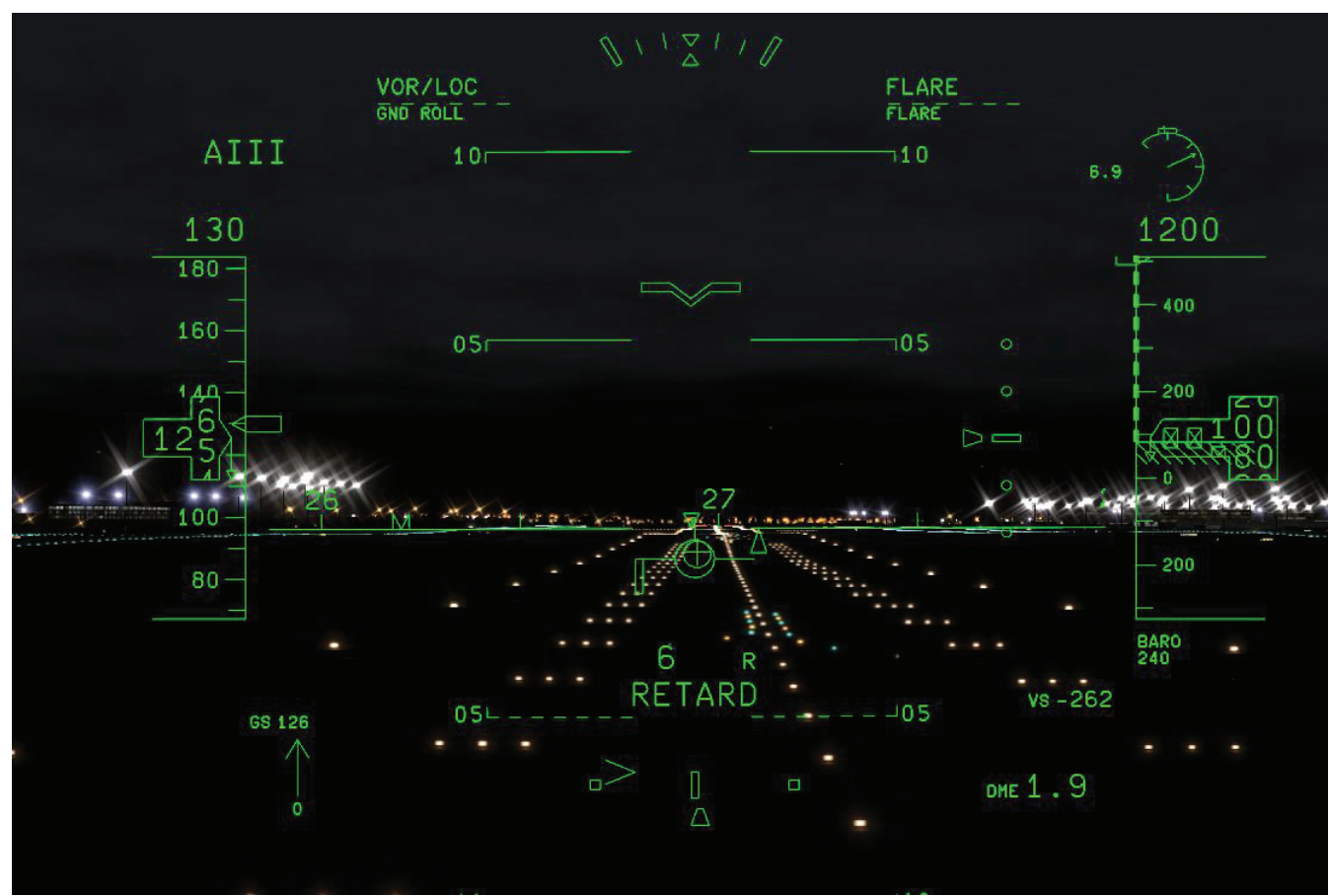

Figure eight: Flare and power retard

\section{Data Sets}

Approach runs were repeated many times to build a significant data set table as shown in figure nine.

\begin{tabular}{|c|c|c|c|r|r|r|r|}
\hline \multicolumn{7}{|c|}{ Landing Data } \\
\hline VCas & \multicolumn{1}{|c|}{ VSI } & X & Y & \multicolumn{1}{|c|}{ Roll } & Pitch & Yaw & \multicolumn{1}{c|}{ YDot } \\
\hline 129.175 & -3.55444 & 1856.55 & -16.4594 & 0.903417 & 2.61301 & 268.106 & -217.109 \\
\hline 125.626 & -6.13441 & 1501.07 & -13.3515 & 0.280554 & 4.30009 & 269.545 & -212.616 \\
\hline 128.963 & -4.63505 & 1417.97 & -10.8708 & -0.79683 & 3.16601 & 268.411 & -217.746 \\
\hline 129.122 & -2.8854 & 1699.15 & -5.10395 & 1.13979 & 2.61905 & 269.663 & -218.568 \\
\hline 130.911 & -2.54083 & 1775.16 & -6.24758 & 0.305645 & 2.64659 & 269.927 & -220.889 \\
\hline 128.647 & -1.90321 & 1614.52 & -7.73683 & 0.182736 & 4.87553 & 269.302 & -217.339 \\
\hline 125.134 & -4.47832 & 1598.42 & -18.6139 & 1.14321 & 3.95532 & 269.579 & -211.657 \\
\hline 128.524 & -2.46431 & 1749.66 & -13.6749 & -0.10052 & 4.40436 & 269.831 & -217.157 \\
\hline 135.607 & -7.55055 & 1178.94 & -7.05621 & -1.26642 & 2.04529 & 269.406 & -228.299 \\
\hline
\end{tabular}

Figure nine: Sample of a data table. Approach speed, vertical speed, lateral and horizontal position at touchdown, roll angle Pitch angle Yaw and vertical acceleration were typically captured as shown. Overall, more than 1,000 runs were required at a variety of airports under a variety of conditions to capture a suitably diverse data set. 


\section{Data Analysis}

Sequential analysis was carried out continuously during testing as the data was gathered to ensure that the system was behaving in a convergent manner and that there were no problems.

If there were, then the cause would need to be investigated before proceeding.

Figure ten shows how this process was applied in the first few hundred runs. To be acceptable the plots must lie below the orange line, which is conformed.

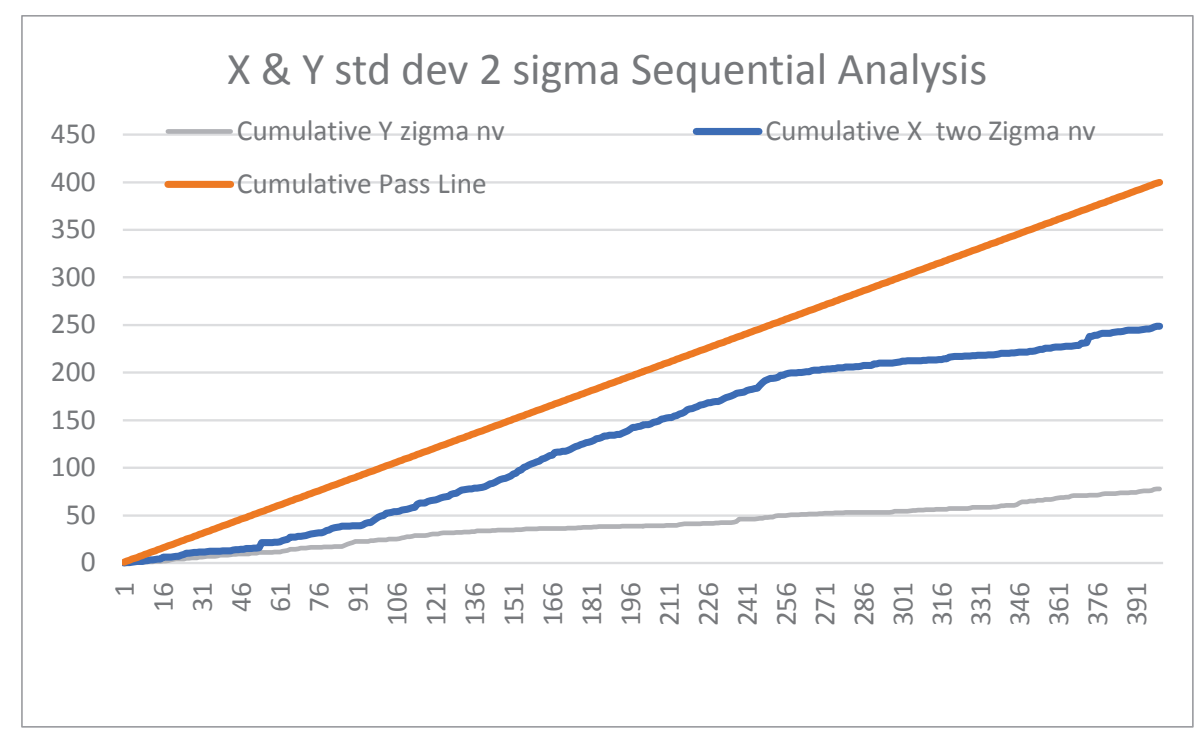

Figure ten: $X \& Y$ std dev

We will recall that performances required to be verified were as follows:

- Longitudinal touch down earlier than a point on the runway $200 \mathrm{ft}$. from the threshold to a probability of $10^{-6}$

- Longitudinal touchdown beyond $2700 \mathrm{ft}$. from threshold to a probability of $10^{-6}$

- Lateral touchdown with the outboard landing gear more than $70 \mathrm{ft}$. from runway centreline to a probability of $10^{-6}$

- Structural limit load, to a probability of $10^{-6}$.

- An acceptable means of establishing that the structural limit load is not exceeded is to show separately and independently that the limit load that results from a sink rate at touchdown is not greater than 10 f.p.s.

- Bank angle resulting in hazard to the airplane to a probability of $10^{-7}$.

- A hazard to the airplane is interpreted to mean a bank angle resulting in any part of the wing, high lift device, or engine nacelle touching the ground

- Also, specific precision must be met on approach as follows

- Glideslope tracking between 700 and 100 feet; the greater of $\pm 35 \mu \mathrm{A}$

○ Localiser tracking between 700 and $300 \mathrm{ft} ; \pm 35 \mu \mathrm{A}$

- Localiser tracking between 300 and $100 \mathrm{ft} ; \pm 25 \mu \mathrm{A}$

- Airspeed tracking between 700 and $50 \mathrm{ft} ; \pm 5$ knots

Examples of this are shown.

Figure eleven illustrates plots of Vertical Speed Indication deviation with $99 \%$ confidence Intervals which show that the results are within the limits expected. 


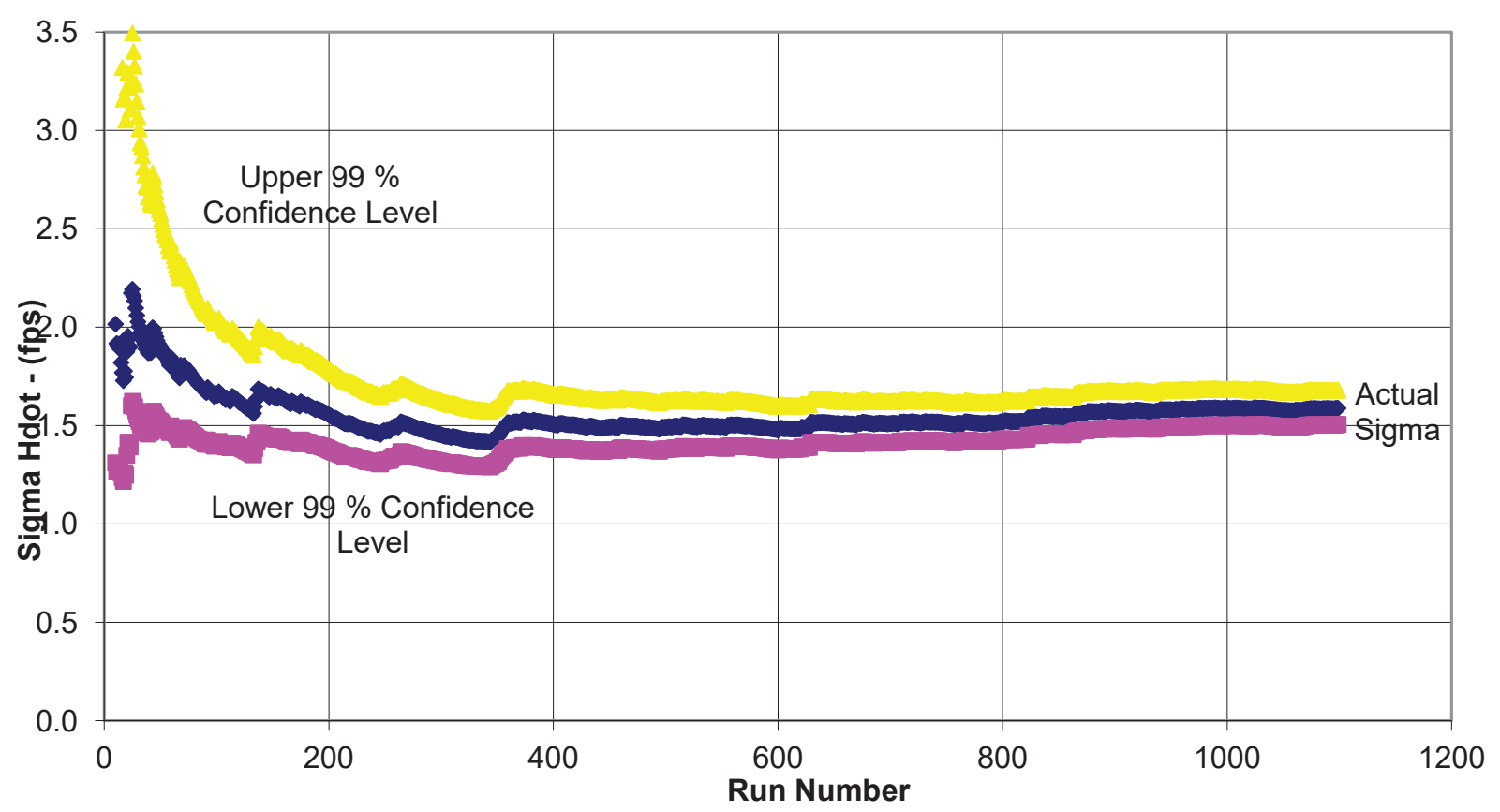

Figure eleven: Vertical speed indication deviation with 99\% Confidence Intervals

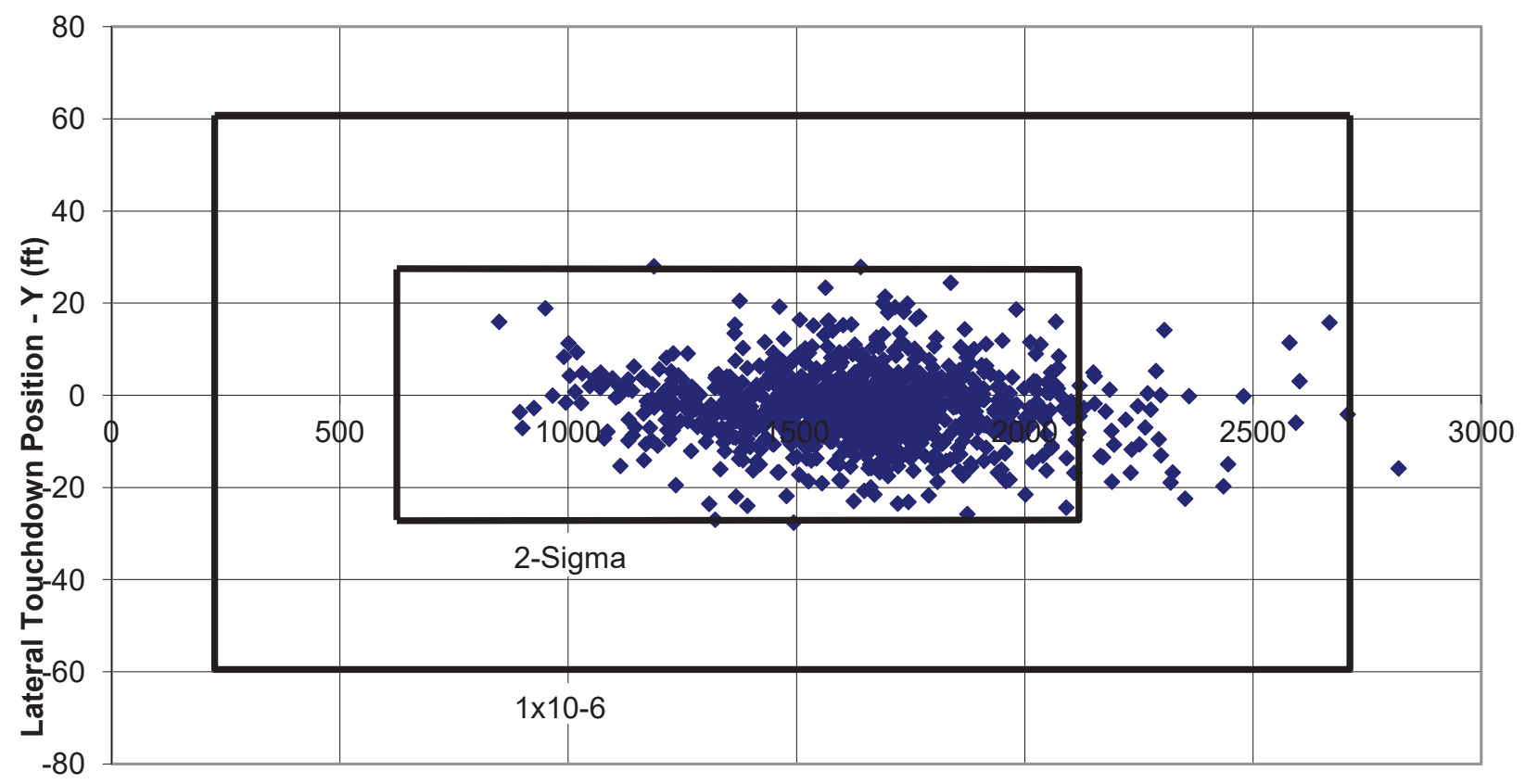

Longitudinal Touchdown Position - X (ft)

Figure twelve: Touchdown footprint. In this example, a bias towards a longer landing was noted caused by a simulation error. This was subsequently corrected. 


\section{Monte Carlo Test Sinkrate Profile}

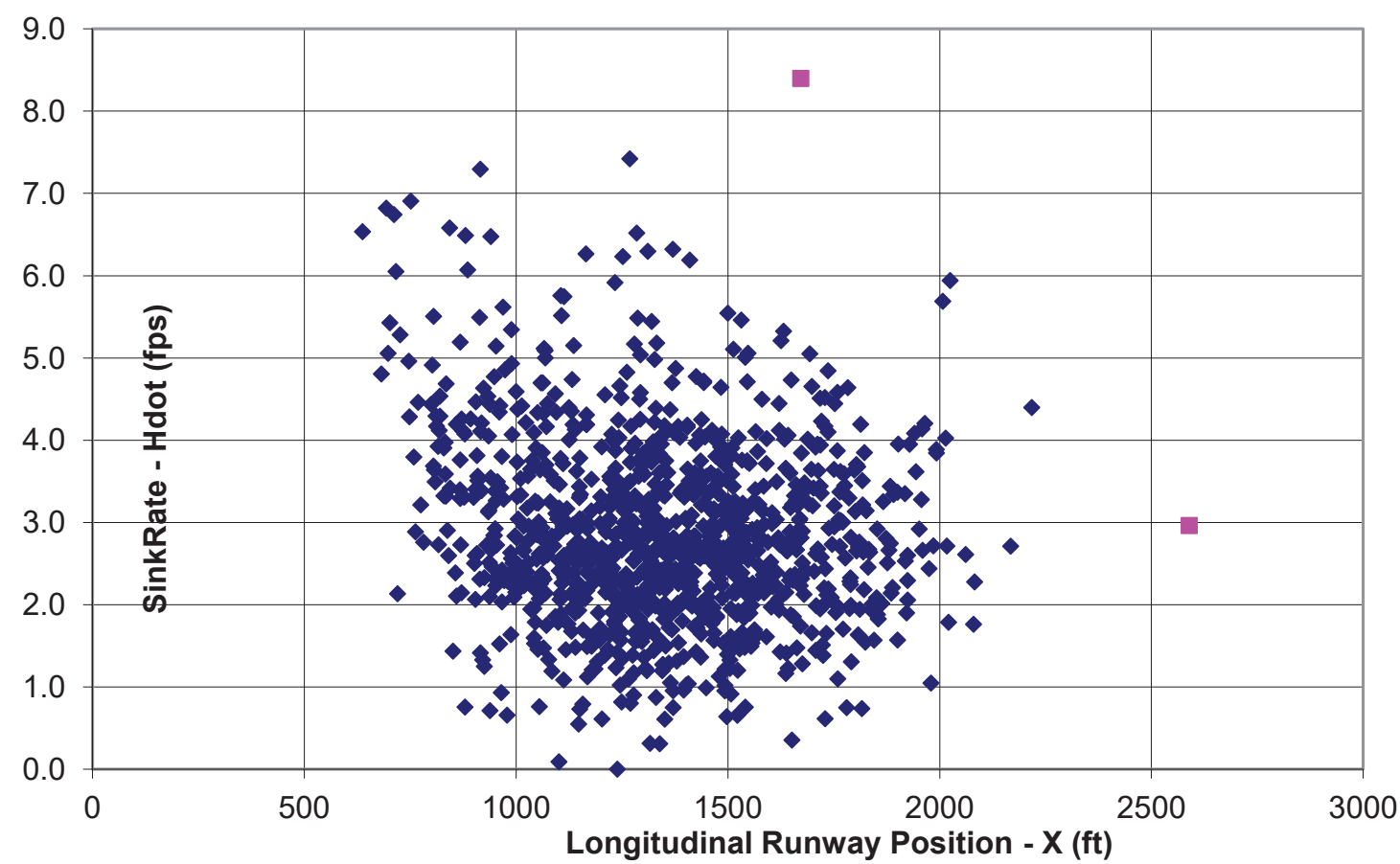

Figure thirteen: Sink rate at touchdown

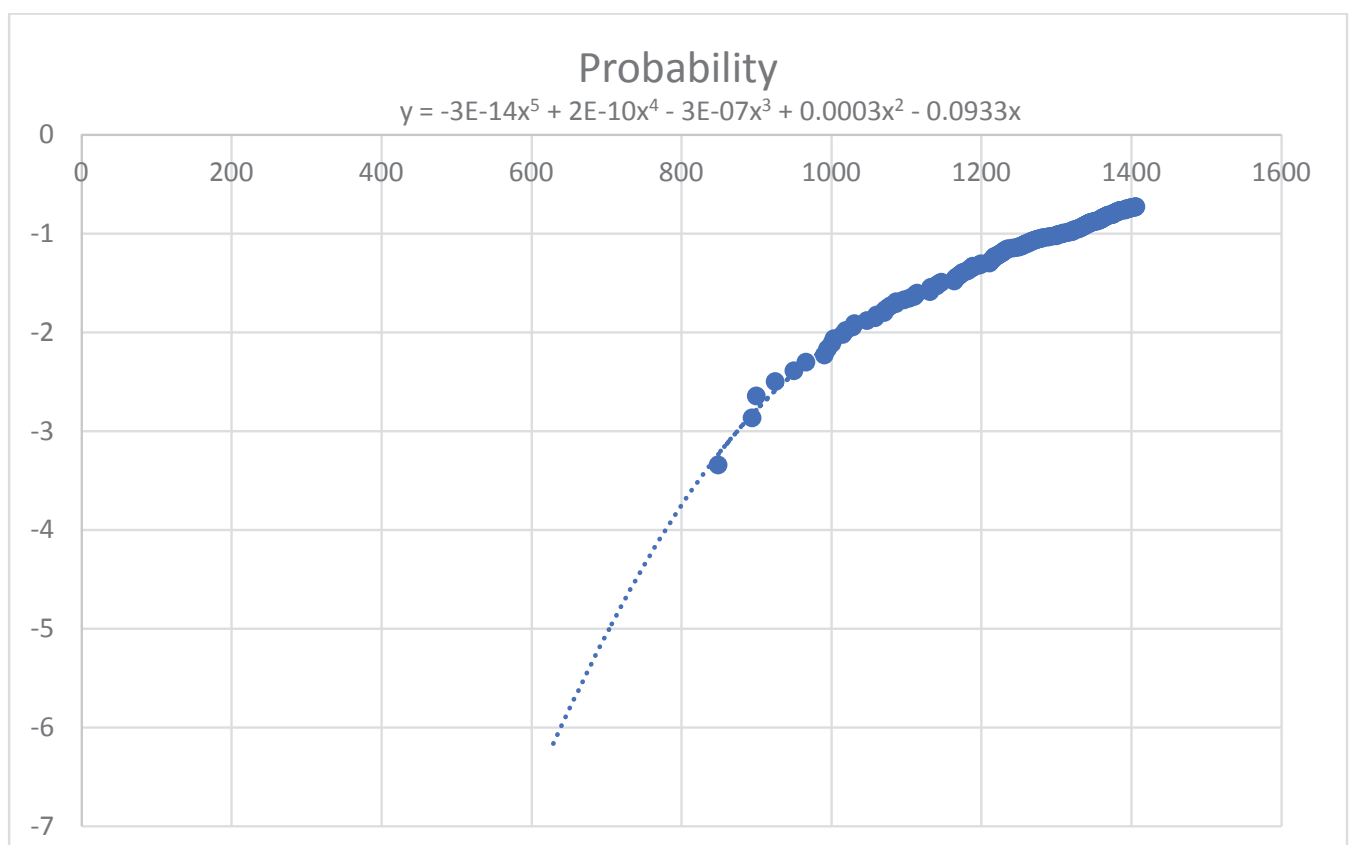

Figure fourteen: Probability of landing less than 200ft from threshold at a probability of $10^{-6}$. As may be appreciated, the $10^{-6}$ value indicated is some 620 feet. 


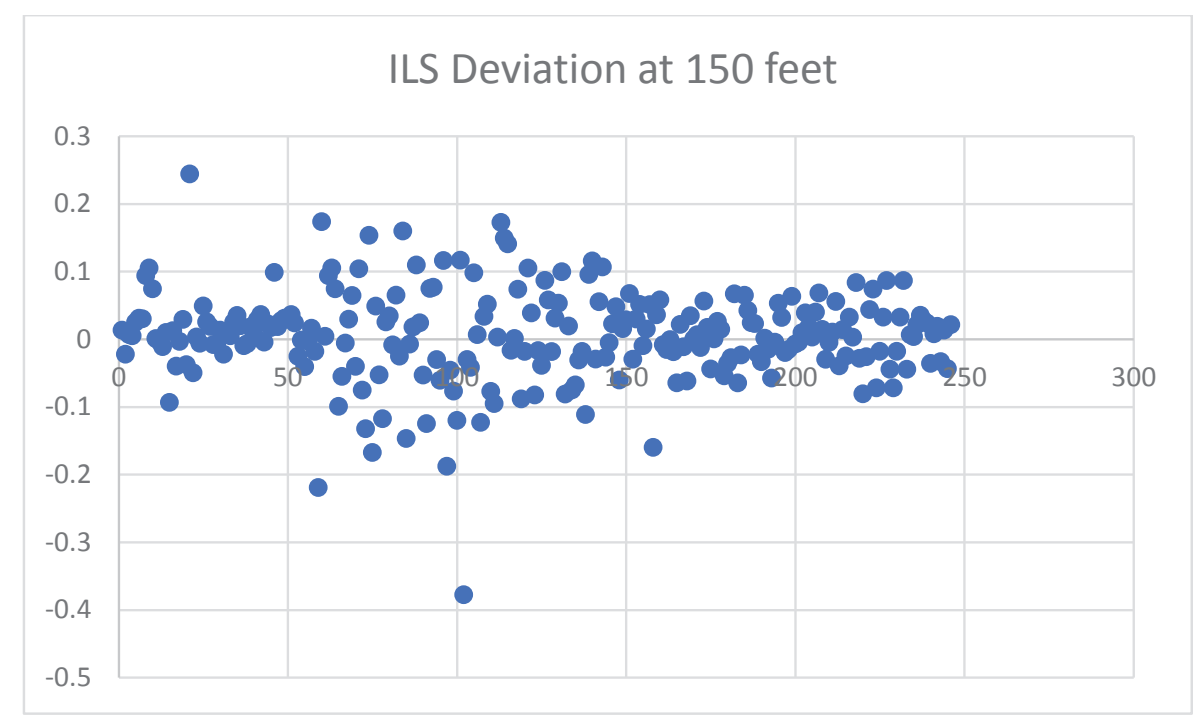

Figure fifteen: ILS tracking accuracy example

The application of "twinning" is a very powerful technique to predict the performance of non-deterministic systems where a probability distribution is unknown and must be investigated by trial.

It should be noted that the system approach described is an engineering development tool to enable correct implementation of the desired system.

For certification purposes, more formal arrangements would need to be employed.

The system also includes extremely effective diagnostic capabilities which are beyond the scope of this paper to describe, both in terms of fault analysis and dynamic adjustment of performance "on the fly".

Hoh Aeronautics

\section{Acknowledgements}

Ansys

Chris Taylor, Simon Bracken, and Darren Guscott of AAL whose invaluable work made all this possible.

Professor Ken Edwards for his valuable inputs

\section{References}

[1] Paul Wisely. 'A digital head-up display system as part of an integrated autonomous landing system concept' Proc. SPIE. 6957, Enhanced and Synthetic Vision 2008 